\title{
Mach Disk Shape in Truncated Ideal Contour Nozzles
}

\author{
R. Stark and B. Wagner \\ German Aerospace Center DLR, Institute of Space Propulsion \\ Langer Grund, D-74239 Hardthausen (Germany)
}

Summary. DLR Lampoldshausen carried out cold flow tests to study the flow field in a truncated ideal contour nozzle at low nozzle pressure ratios. A convex shaped Mach disk was found for nozzle pressure ratios less than 10 and a slight concave one for nozzle pressure ratios more than 20. Due to boundary layer transition the convex Mach disk is temporary tilted and redirects the flow towards the nozzle wall. Accompanying numerical simulations compare well with the experiments.

\section{Introduction}

A series of CFD studies and tests went in for separated nozzle flows in the past. A number of tests showed a significant side load peak for low nozzle pressure ratios (NPR). DLR Lampoldshausen studied this peak [6] experimentally and identified a re-attached flow as an origin. This flow condition was present in thrust optimized parabolic (TOP) nozzles as well as in truncated ideal contour (TIC) nozzles. Re-attached flows are well-known for TOP nozzles where the so called cap shock pattern redirects the flow towards the wall. A competitive CFD study [6] for a TIC nozzle yield a concave bowed Mach disk creating a flow pattern comparable to this cap shock pattern. This bowed mach disk was thought to explain at low NPR a partial redirected flow towards the nozzle wall.

Bowed Mach disks at moderate NPRs could also be found in computations from Nasuti et al. [9] and Pilinski [10]. Frey et al. [5] show a Schlieren image where a shortened TIC nozzle features such a kind of Mach disk. These experimental and numerical studies as well as an intensive discussion within Europe's Flow Separation Control Device Group (FSCD) induced a test campaign studying the Mach disk in TIC nozzles in more detail.

\section{Test Setup}

The tests were conducted on the horizontal cold flow test position P6.2 using dry nitrogen as a fluid. The maximum total pressure and the minimum total temperature were $5.6 \mathrm{MN} / \mathrm{m}^{2}$ and $230 \mathrm{~K}$, respectively. The flow passes a settling chamber, a cross-section constriction, and a bending tube section before it accelerates in the convergent-divergent nozzle to supersonic velocity (Figure 1). As the flow pattern at low NPR $(<10)$ were of interest, where oblique shock and Mach disk are located inside the nozzle, an acrylic glass TIC nozzle was cut step by step from $L / R^{*}=12$ down to $L / R^{*}=1.75$. It was equipped with up to 50 pressure transducer ports in stream wise direction with a spacing of $2.5 \mathrm{~mm}$ each, starting in the nozzle's throat. The machine-made contour was checked in 3 axial planes and the deviation compared to the design was less than $5 \mu \mathrm{m}$. The exhaust jet was studied with a Color Schlieren setup based on the dissection technique developed by 


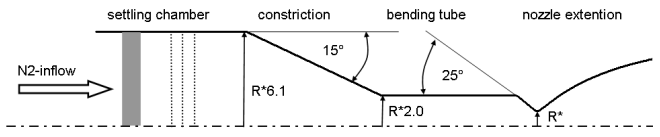

Fig. 1. Sketch of horizontal test section

Cords [2] and improved by Ciezki [1]. Nozzle and test setup are described in more detail in Frey [4] and Stark [13].

\section{Discussion of Experimental Results}

The obtained flow patterns can be compared to study the influence of the nozzle length on separation location, back pressure, and finally the position and the shape of the Mach disk.

Figure 2a gives the separation locations of all tested nozzle lengths. The data follow a
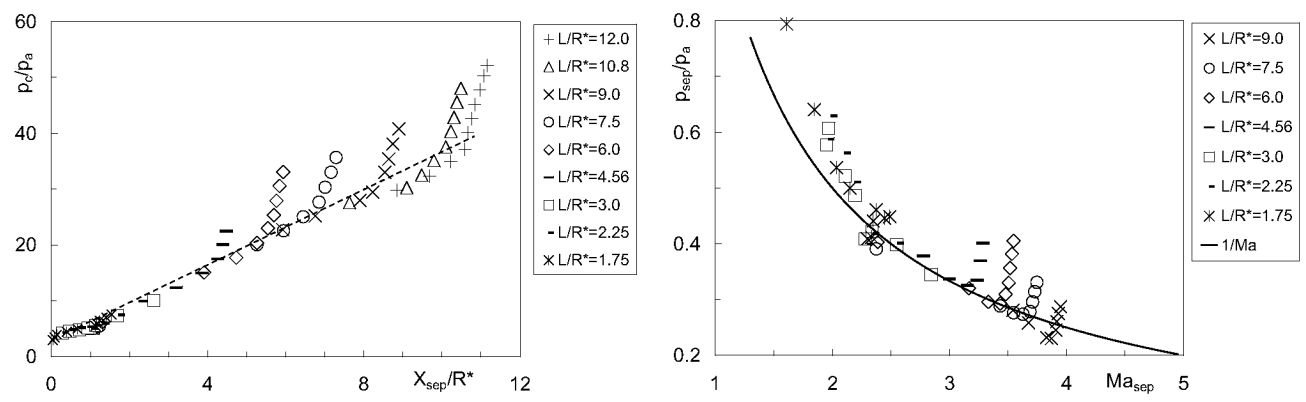

Fig. 2. Averaged separation location. (a) Normalized nozzle coordinates versus NPR $\left(p_{c} / p_{a}\right)$. (b) Separation and wall Mach number

linear trend as long as the flow really separates. If the separation zone reaches the nozzle lip, a continued NPR rise only results in a compression of the separation zone, indicated by a distinct gradient change. The discontinuities around NPR $=30$ are due to nitrogen condensation resulting in a delayed separation. Thus an influence of the nozzle length on the separation location can be neglected. Figure $2 \mathrm{~b}$ is a more conventional plot without condensation affected data where the separation pressure (lowest wall pressure) is given as a function of the wall Mach number. The criteria

$$
p_{\text {sep }} / p_{a}=1 / M a_{\text {sep }}
$$

reproduces the separation pressure data for wall Mach numbers $>2.5$ quite well. This trend gets lost for wall Mach numbers $<2.25$. The intermediate range around a wall Mach number of 2.4 (representing low NPR of 5) is unstable.

Figure 3a gives the related pressure coefficients as a function of the Reynolds number after Donaldson $[3,12,7]$, who studied supersonic tube flows, where $p_{2}$ is the pressure 

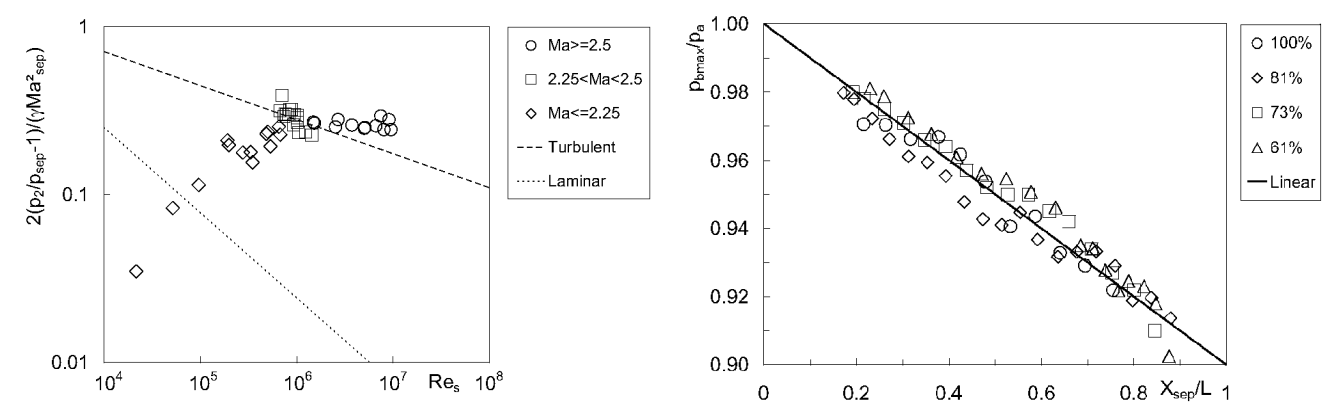

Fig. 3. (a) Pressure coefficient and Reynolds number. (b) Averaged maximum back pressure

downstream the shock and $\gamma$ the adiabatic exponent. Only condensation free data were taken into account. Included are critical pressure coefficients for turbulent $\left(\sim R e_{s}^{-0.2}\right)$ and laminar $\left(\sim R e_{s}^{-0.5}\right)$ separation on flat plates. The squares represent the unstable Mach number region around 2.4. With Reynolds numbers about $10^{6}$, known for laminarturbulent transition [11], they split up the remaining data in two distinct zones. Separation data for Mach numbers $>2.5$ (circles) show a clearly turbulent separation behaviour. The data for Mach numbers $<2.25$ (diamonds) a more laminar like. Therefore the separation criteria (1) is valid for turbulent flows.

Figure $3 \mathrm{~b}$ shows a linear correlation between the maximum pressure inside the separated back flow region $p_{b \max } / p_{a}$ and the separation location. Compared are results of the several times shortened nozzle $(61 \%, 73 \%$ and $81 \%$ of initial length). The effective length of the nozzle is hereby negligible and the linear trend of $p_{b \max } / p_{a}$ converges to 0.9 , corresponding with the full flowing nozzle. This is to say: The oblique separation shock re-compresses the flow to $\sim 90 \%$ of the ambient pressure and the remaining adaption to ambient conditions takes place over the free jet.


Fig. 4. Schlieren Images (a) $L / R^{*}=9 ; \quad \mathrm{NPR} \simeq 30 ; \quad H_{\text {Mach }}=1.7 \mathrm{~mm} . \quad$ (b) $L / R^{*}=1.75 ;$ $\mathrm{NPR} \simeq 5.5 ; H_{\text {Mach }}=1.8 \mathrm{~mm}$ 
In opposite to CFD studies $[6,9,10]$ where clearly bowed concave Mach disks at moderate NPRs were predicted, the Schlieren images only show Mach disks with a slight curvature limited to the centre (Figure 4a). Figure 5a gives the directionless height of the curvature related to the unaffected base as a function of NPR. A maximum is reached for a NPR of 30 to 35. This distribution correlates with the side loads measured for the TIC nozzle of full length by Frey [4] as shown in the graph. A coherence might be given, especially if a vortex is trapped downstream the Mach disk. A second peak is given for NPR below 10. But here the former concave shape turns to a convex one (Figure 4b). Therefore a concave bowed Mach disk at low NPR, as obtained by former CFD studies [6], must be excluded as the origin of a redirected flow towards the wall resulting in side loads.


Fig. 5. (a) Averaged Mach disk curvature $\left|H_{M a c h}\right|$. (b) Tilted Mach disk; $L / R^{*}=1.75$; $\mathrm{NPR}=4.93$

The pressure coefficients (Figure 3a) show that a re-laminarised boundary layer is present in the nozzle throat. Therefore the separation zone has to pass, during transient start-up and shut-down, through the transition to turbulence again. Its performance changes and the position is abruptly shifted downstream. This process is circumferentially asymmetrical and results in a temporary tilted Mach disk, shown in Figure 5b. In nozzles of full length this tilted Mach disk redirects the flow towards the wall. This is the reason of the partial re-attachment at low NPR causing huge side loads.

This process is locally limited and reversible as the NPR is further increased. It is a close-limited instability in separation behaviour.

\section{CFD-Analysis}

The Tau-code, developed by DLR [8], was used for accompanying computations. The flow field inside and outside of the nozzle was simulated on axisymmetric hybrid grids. The boundary layer of the adiabatic nozzle wall was resolved by a structured grid consisting out of 30 layers and a $y+$ value less then 1 . The simulated inflow conditions are summarised in Table 1 and they correspond to the experiments shown in Figure $4 \mathrm{~b}$. This case was chosen due to the extrem downstream bended Mach disk, the coincidence with the side load peak and former computations of Kwan [6] where a reattached flow 
could be observed at low NPRs. The boundary conditions are completed with a farfield interpolation based on the ambient conditions during the experiment.

\begin{tabular}{clcc}
\hline NPR & Simulated gas & $T_{0}[\mathrm{~K}]$ & $T_{a}[\mathrm{~K}]$ \\
\hline 5.5 & Nitrogen & 267 & 288 \\
\hline
\end{tabular}

Table 1. Boundary conditions for the simulations according to the experiments

For the given flow conditions the AUSMDV upwind solver with a second order MUSCL gradient reconstruction was applied. The temporal gradients are solved by a three stage Runge-Kutta scheme. Due to the acceptable performance on separated nozzle flows the Spalart Allmaras turbulence model was chosen for the computations.

Residual smoothing was used for a better convergence behavior. The shock pattern and the separated flow at the nozzle wall were achieved on a very coarse grid. Starting from that initial grid and solution, local adaptation was used to refine both the region around the separation point and the Mach disk. The adaptation was successivly continued as described in more detail in Wagner et al. [14]. The development of the wall pressure distribution depending on the grid resolution can be found in Figure 6a starting with the first adapted grid. The results are compared with the measured wall pressures. Due to the minimum of $2.5 \mathrm{~mm}$ between the pressure transducers the exact separation position cannot be determined, but the computed pressure distribution compares well with the present experimental values. The dismatch of the first position at $X / R^{*}=0.28$ is within the accuracy of the implemented sensor.
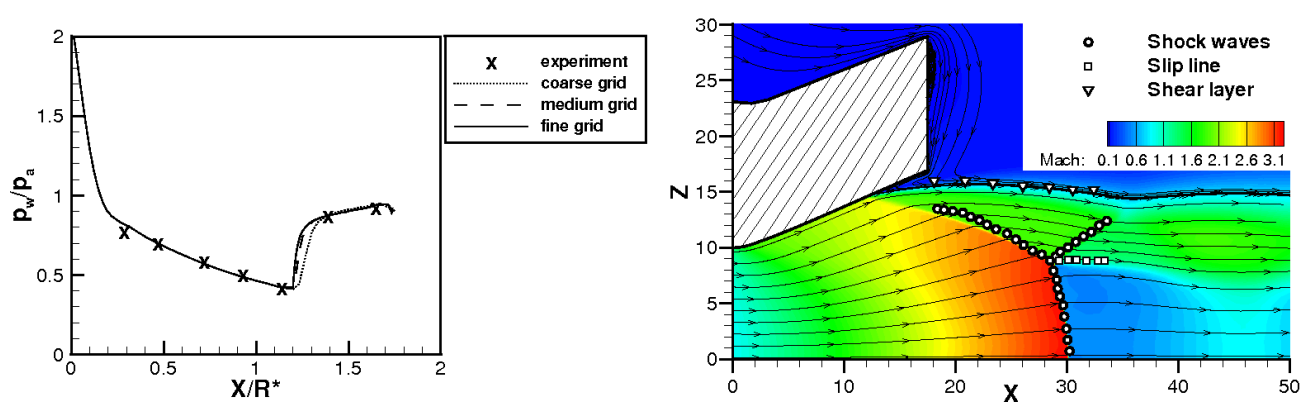

Fig. 6. Numerical Results of the $L / R^{*}=1.75$ nozzle (a) Pressure distribution. (b) Mach number flow field with stream lines compared to experimental data

In Figure $6 \mathrm{~b}$ the numerical Mach number distribution is overlaid by values taken from the Schlieren image $4 \mathrm{~b}$. The shape of the Mach disk and the reflecetd shock are well resolved by the computations. The oblique shock coming from the separation point is more curved in the experiments. Additional to the shock wave position, the trend of both the slip line and the shear layer are extracted from the Schlieren images as far as they were visible and they also compare well with the CFD results.

Both in the experiments and in the computations the Mach disk is bended downstream. The convex shaped Mach disk is the only possability to deflect the divergent flow near the nozzle throat to a parallel flow. 
The authors would like to thank their colleagues of the 'Flow Separation Control Device' group [5] (CNES, DLR, Astrium ST, ESTEC, ONERA, LEA at Portiers, Snecma, Volvo Aero Corporation) for fruitful scientific exchanges.

\section{References}

1. Ciezki H.: Entwicklung eines Farbschlierenverfahrens unter besonderer Berücksichtigung des Einsatzes an einem Stosswellenrohr, Diploma Thesis, Technical University Aachen, 1985

2. Cords P.: A High Resolution, High Sensitivity Colour Schlieren Method, S.P.I.E Journal, Vol. 6, 1968

3. Donaldson C. and Lange R.: Study of the Pressure Rise across Shock Waves Required to Separate Laminar and Turbulent Boundary Layers, NACA RM L52C21, 1952

4. Frey M.: Behandlung von Strömungsproblemen in Raketendüsen bei Überexpansion, $\mathrm{Ph}$. D. Thesis, Universität Stuttgart, 2001

5. Frey M. et al.: Joint European Effort Towards Advanced Rocket Thrust Chamber Technology, 6th International Conference on Launcher Technologies, 2005

6. Kwan W. and Stark R.: Flow Separation Phenomena in Subscale Rocket Nozzles, AIAA Paper 2002-4229, 2002

7. Lawrence R.: Symmetrical and Unsymmetrical Flow Separation in Supersonic Nozzles, NASA CR 92587, 1967

8. Mack A. and Hannemann V.: Validation of the Unstructured DLR-TAU-Code for Hypersonic Flows, AIAA Paper 2002-311, 2002

9. Nasuti F., Onofri M. and Pietropoli E.: Prediction of Shock Generated Vortices in Rocket Nozzles, AIAA Paper 2005-317, 2005

10. Pilinski C. and Nebbache A.: Flow Separation in a Truncated Ideal Contour Nozzle, Journal of Turbulence, Vol. 20, No. 3, 2004

11. Schlichting H.: Grenzschicht-Theorie, 9. Auflage, ISBN 3-540-55744-X, 1997

12. Shapiro A.: The Dynamics and Thermodynamics of Compressible Fluid Flow, Volume 2, ISBN 0-89874-566-7, 1985

13. Stark R. and Wagner B.: Experimental Flow Investigation of a Truncated Ideal Contour Nozzle, AIAA Paper 2006-5208, 2006

14. Wagner B., Karl S. and Hannemann K.: Test Case 1a: Short Nozzle Under Separated Flow Condition Numerical Investigation with the DLR Tau-Code, Proceedings of The FSCD-ATAC Workshop, Noordwijk, 15-16th November, The Netherlands, 2006 